Pesq. Vet. Bras. 37(9):971-976, setembro 2017 DOI: $10.1590 / \mathrm{S} 0100-736 \mathrm{X} 2017000900012$

\title{
Neuromiopatia isquêmica em cinco cães (2008-2016) ${ }^{1}$
}

\author{
Angel Ripplinger ${ }^{2}$, Rafael 0. Chaves ${ }^{2}$, Glaucia D. Kommers ${ }^{3}$, Flavia S. da Luz ${ }^{2}$, Dakir \\ Polidoro $^{2}$, Rafael A. Fighera ${ }^{3}$ e Alexandre Mazzanti ${ }^{4 *}$
}

\begin{abstract}
Ripplinger A., Chaves R.O., Kommers G.D., Luz F.S., Polidoro D., Fighera R.A. \& Mazzanti A. 2017. [Ischemic neuromyopathy in five dogs (2008-2016).] Neuromiopatia isquêmica em cinco cães (2008-2016). Pesquisa Veterinária Brasileira 37(9):971-976. Departamento de Clínica de Pequenos Animais, Universidade Federal de Santa Maria, Avenida Roraima 1000, Camobi, Santa Maria, RS 97105-900, Brazil. E-mail: alexamazza@yahoo.com.br

This study aimed to present epidemiological, clinical and anatomopathological data of five dogs with ischemic neuromyopathy secondary to aortic thrombosis or thromboembolism. The dogs were females, with eleven years of age or more, that had paraplegia $(80 \%)$ or hind limb monoplegia (20\%). Forty percent of the cases $(n=2)$ had lameness previously the motor disfunction. The main clinical findings include absence of unilateral or bilateral femoral pulse, cool hind limbs extremities, muscle pain above the knee joint and absence of nociception (deep pain) on the hind limbs digits. Four dogs were euthanized due to the poor prognosis, and the last one died within 24 hours of hospital admission. The necropsy findings were cardiac lesions in three dogs, neoplastic disease in one, and unknown cause on the other. The thrombi were located on the abdominal aorta, extending to the iliac arteries. Even with low incidence, the ischemic neuromyopathy should be included in the differential diagnosis of dogs with lameness, paraparesis, or unilateral or bilateral paraplegia, when neurological signs are compatible with lower motor neuron lesion (L4-S3) and acute or chronic progression. The digital and/or vascular doppler evaluation of the femoral pulse must be used and can assist on the disease diagnosis, avoiding treatments for other illnesses.
\end{abstract}

INDEX TERMS: Aorta, thromboembolism, canine, thrombus, ischemic neuromyopathy.

RESUMO.- 0 objetivo deste estudo foi apresentar dados epidemiológicos, clínicos e anatomopatológicos de cinco cães com neuromiopatia isquêmica secundária a trombose ou tromboembolismo aórtico. Os cães eram fêmeas, com idade $\geq 11$ anos, que apresentavam paraplegia (80\%) ou monoplegia (20\%) de membro pélvico. Em $40 \%$ dos casos $(n=2)$ foi relatada claudicação previamente à disfunção motora. Os principais achados clínicos foram ausência de pulso femoral uni ou bilateral, extremidade de membros pélvicos frias, dor muscular acima da articulação do joelho e ausência de nocicepção (dor profunda) nos dígitos dos membros pélvicos.

\footnotetext{
${ }^{1}$ Recebido em 4 de agosto de 2016.

Aceito para publicação em 25 de janeiro de 2017.

2 Programa de Pós-Graduação em Medicina Veterinária, Universidade Federal de Santa Maria (UFSM), Av. Roraima 1000, Santa Maria, RS $97105-$ 900, Brasil.

${ }^{3}$ Departamento de Patologia, Centro de Ciências da Saúde, UFSM, Av. Roraima 1000, Santa Maria, RS 97105-900, Brasil.

${ }^{4}$ Departamento de Clínica de Pequenos Animais, Centro de Ciências Rurais, UFSM, Av. Roraima 1000, Camobi, Santa Maria, RS 97105-900, Brasil. *Autor para correspondência: alexamazza@yahoo.com.br
}

Quatro cães foram submetidos à eutanásia devido ao prognóstico desfavorável e um veio a óbito em menos de 24 horas de internação. Na necropsia foi encontrada alteração cardíaca em três cães, neoplásica em um e, sem causa determinante em outro caso. Os trombos estavam localizados na aorta abdominal e se estendiam até as artérias ilíacas. Mesmo sendo baixa a incidência, a neuromiopatia isquêmica deve ser incluída no diagnóstico diferencial de cães com claudicação, paraparesia ou paraplegia uni ou bilateral, quando os sinais neurológicos são compatíveis com lesão de neurônio motor inferior (L4-S3) e de evolução aguda ou crônica. A avaliação do pulso femoral digital e/ou por doppler vascular deve ser empregada e pode auxiliar no diagnóstico da doença evitando que os animais iniciem tratamento para outras afecções.

TERMOS DE INDEXAÇÃO: Aorta, tromboembolismo, canino, trombo, neuromiopatia isquêmica.

\section{INTRODUÇÃo}

A neuromiopatia isquêmica é uma síndrome clínica secundária à interrupção do aporte sanguíneo (gerando isque- 
mia) geralmente para os membros pélvicos, causada pela presença de trombo na trifurcação da aorta abdominal (Dewey \& Talarico 2016). Esse material pode ser formado pela deposição intravascular de fibrina e plaquetas localmente ou ser secundário a um tromboembolismo (Smith \& Tobias 2004). A formação de trombo pode ocorrer devido a distúrbios em um ou mais fatores como alteração no fluxo sanguíneo, integridade endotelial e coagulação/fibrinólise, também conhecidos como tríade de Virchow (Rosenberg \& Aird 1999, Boswood et al. 2000).

A isquemia pode causar paresia ou paralisia tanto em felinos (Smith et al. 2003, Smith \& Tobias 2004) como em caninos (Boswood et al. 2000, Winter et al. 2012). Em felinos a fisiopatologia está relacionada, principalmente, à doença cardíaca em que um trombo se forma no lado esquerdo do coração e dele se desprende um tromboêmbolo que causa a obstrução aórtica (Smith \& Tobias 2004). Em caninos é uma afecção considerada rara (Winter et al. 2012) e são reconhecidas diversas doenças que podem predispor à trombose e ao tromboembolismo aórtico como hiperadrenocorticismo, glomerulopatias graves, doença cardíaca, neoplasma (Van Winkle et al. 1993, Boswood et al. 2000, Gonçalves et al. 2008) e ainda mecanismos locais de formação de trombo (Lake-Bakaar et al. 2012, Winter et al. 2012).

Alguns sinais clínicos inespecíficos da neuromiopatia isquêmica como claudicação (Kim \& Park 2012, Boswood et al. 2000), paresia ou plegia, diminuição de reflexos espinhais e do tônus muscular (Gonçalves et al. 2008) podem levar o clínico a suspeitar de afecção de origem ortopédica ou neurológica (Kim \& Park 2012, Gonçalves et al. 2008), dificultando o diagnóstico da doença.

Devido a isso, o objetivo do presente trabalho foi relatar dados epidemiológicos, clínicos e anatomopatológicos de cinco cães com neuromiopatia isquêmica secundária à trombose ou tromboembolismo aórtico e apresentar os principais fatores que podem auxiliar na diferenciação de outras doenças que causam sinais clínicos semelhantes.

\section{MATERIAL E MÉTODOS}

Foram revisados os registros médicos do serviço de neurologia de cães que apresentavam alterações nos membros pélvicos (claudicação, paresia e plegia) entre 2008 e 2016, atendidos no Hospital Veterinário da Instituição e incluídos aqueles com diagnóstico clínico e pós-morte de trombose ou tromboembolismo aórtico.

Foram compilados dados epidemiológicos (idade, sexo, raça, evolução do quadro, motivo do óbito/eutanásia), bem como dados clínicos (sinais clínicos, suspeita clínica, motivo de encaminhamento para avaliação neurológica, alterações no exame neurológico e tratamento), de exames complementares laboratoriais (creatinoquinase), exame de doppler da artéria femoral e dos laudos de necropsia para localização do trombo e a possível origem deste.

A evolução do quadro foi classificada em aguda, para os casos em que os sinais clínicos estavam em um período inferior a 24 horas, ou crônica, superior a este período.

Das 515 fichas neurológicas revisadas, foram incluídos, nesse estudo, cinco cães com neuromiopatia isquêmica secundária a trombose ou tromboembolismo aórtico.

\section{RESULTADOS E DISCUSSÃO}

A neuromiopatia isquêmica secundária a tromboembolismo é descrita em felinos com apresentação aguda de pa- resia ou paralisia de membros pélvicos, dor, ausência de pulso femoral, leito ungueal cianótico e ausência de dor profunda (Laste \& Harpster 1995, Schoeman 1999, Smith et al. 2003). 0 prognóstico nessa espécie é desfavorável e a recorrência é alta, com a taxa de eutanásia chegando a 35\% dos casos (Smith et al. 2003). Já em cães, informações clínicas sobre trombose ou tromboembolismo aórtico são menos documentadas na literatura (Lake-Bakaar et al. 2012) e a maioria dos trabalhos recentes são relatos de casos isolados, sendo mais escassos os dados na literatura nacional (Marcasso et al. 2011, Diamante et al. 2016).

No presente estudo, a doença ocorreu em cadelas (100\% dos casos), com idade entre 11 e 15 anos, sendo três de raça pura (dois Teckel e um Poodle) e dois sem raça definida (Quadro 1). Entretanto, estudos com maior número de pacientes envolvidos comprovaram a ausência de predileção por sexo, raça ou idade, incluindo pacientes a partir de cinco meses de idade (Van Winkle et al. 1993). Os dados epidemiológicos, clínicos, laboratoriais e de necropsia estão representados no Quadro 1.

A ocorrência desta afecção em caninos atendidos no serviço de neurologia do Hospital Veterinário da Instituição foi considerada baixa $(0,97 \%)$, assim como em outros hospitais veterinários (Lake-Bakaar et al. 2012). Um dos motivos a ser considerado para as baixas casuísticas é provavelmente a falta ou diagnóstico equivocado, pois a apresentação clínica e a patogênese da doença em cães diferem daquelas que ocorrem em felinos (Winter et al. 2012), além de ser uma doença pouco comentada na espécie em questão.

Quanto à evolução clínica da doença, em três pacientes (60\%) foi crônica ( $>24$ horas), que progrediu para paraplegia em dois e monoplegia em um caso e, dois (40\%) de forma aguda, caracterizada por paraplegia. Dois pacientes (40\%), com evolução acima de 24 horas apresentaram claudicação em apenas um dos membros pélvicos precedendo a disfunção motora bilateral. A claudicação pode ser relatada provavelmente pela obstrução incompleta do fluxo sanguíneo e a dor muscular decorrente da isquemia parcial do membro pélvico (Lake-Baakaar et al. 2012). Supõe-se que uma parte desses pacientes evolui para paralisia secundária à obstrução total do fluxo sanguíneo arterial para os membros pélvicos, como ocorreu com dois dos cães do presente estudo (cães 3 e 4, Quadro 1), quando não é iniciado tratamento adequado precocemente. Apesar de serem descritas em cães, tanto a apresentação aguda como a crônica, a primeira está associada a sinais clínicos mais severos e menor sobrevida (Gonçalves et al. 2008, Lake-Baakaar et al. 2012). Essa diferença entre a sobrevida e a gravidade dos sinais quanto aos dois tipos de apresentação clínica não pode ser observado nos pacientes do presente estudo provavelmente pelo fato de, nos casos crônicos os pacientes (dois deles) já terem sido levados para atendimento anterior em que o quadro foi confundido com doença medular, retardando uma tentativa de tratamento específico para a afecção vascular em questão.

A raça dos cães pode influenciar o clínico a suspeitar de lesão medular, principalmente quando envolve aquelas condrodistróficas como o Teckel, situação encontrada em dois cães deste estudo (Quadro 1). Para casos crônicos du- 
vidosos, a avaliação da marcha (quando o paciente ainda não está em quadro plégico) pode auxiliar na diferenciação da afecção medular (segmento L4-S3), pois no envolvimento medular a paresia geralmente vem acompanhada de ataxia proprioceptiva (Thomas 2000, Goldstein 2010).

Neste estudo, foram encontradas extremidades dos membros pélvicos frias em todos dos casos ( $n=5)$, temperatura retal abaixo do fisiológico em quatro, pulso femoral ausente unilateralmente em um e bilateralmente em quatro casos. Dois pacientes apresentaram lesão necrótica nos coxins e outro com necrose muscular, identificada pela coloração escurecida da região ao exame clínico (Fig.1A) e evidenciada na necropsia após a remoção da pele (Fig.1B).

Em felinos com neuromiopatia isquêmica, a temperatura retal abaixo do fisiológico é associada ao prognóstico desfavorável, provavelmente pelo fato de representar baixa perfusão sistêmica e até mesmo choque (Smith et al. 2003). Em cães, no entanto, esse achado ainda não é considerado fator prognóstico (Boswood et al. 2000, Gonçalves et al. 2008), possivelmente pelos poucos estudos da neuromiopatia isquêmica realizados nesta espécie.

A avaliação da locomoção/marcha durante exame neurológico revelou paraplegia em quatro cães e monoplegia associada à monoparesia contralateral em um cão e sem ataxia proprioceptiva. As alterações foram assimétricas em um cão e simétricas nos demais $(n=4)$. Em todos os pacientes foram observadas deficiências proprioceptivas, com reflexos segmentares espinhais ausentes bilateralmente em quatro cães e, em um cão, ausente no membro pélvico esquerdo e diminuído no direito. 0 tônus muscular estava diminuído em todos os casos. Verificou-se ausência de dor profunda bilateral $(n=4)$ ou unilateral $(n=1)$, além de ausência de dor a palpação epaxial em todos os casos. Esses achados clínicos decorrentes da lesão isquêmica (Dewey \& Talarico 2016) podem confundir o clínico no momento da avaliação do paciente em suspeitar de doenças que afetam a medula espinhal entre os segmentos L4-S3 (Gonçalves et al. 2008), já que os sinais são semelhantes.

Uma das maneiras que permite reduzir ou até mesmo descartar o envolvimento medular é a presença de sensibilidade e mesmo dor grave acima da articulação do joelho, evidenciada na neuromiopatia isquêmica. Por outro lado, nos casos de paraplegia sem dor profunda de origem medular ocorre perda da sensibilidade em todo o membro do paciente. Assim, é prudente testar a sensibilidade acima do joelho em cães paraplégicos sem dor profunda quando os reflexos segmentares indicam lesão entre L4-S3, mesmo sendo baixa a ocorrência dessa doença vascular.

0 principal achado laboratorial foi aumento acentuado na dosagem da enzima creatinoquinase (CK) como evidenciado no Quadro 1, considerando os valores de referência menor que 125UI/L (González \& da Silva 2006). A CK é a enzima muscular mais comumente utilizada para avaliação de dano muscular, pois é uma enzima citosólica liberada para o exterior quando os miócitos são danificados

Quadro 1. Dados epidemiológicos, dos exames neurológicos, clínicos, laboratoriais e de necropsia dos cães com neuromiopatia isquêmica

\begin{tabular}{|c|c|c|c|c|c|c|c|c|c|}
\hline Raça & Sexo & $\begin{array}{l}\text { Idade } \\
\text { (anos) }\end{array}$ & $\begin{array}{l}\text { Evolução } \\
\text { clínica }\end{array}$ & Exame neurológico & Exame clínico geral & $\begin{array}{l}\text { Temperatura } \\
\text { retal }\end{array}$ & $\begin{array}{l}\text { Valores } \\
\text { de CK }\end{array}$ & $\begin{array}{c}\text { Provável causa } \\
\text { do trombo }\end{array}$ & Achados necropsia \\
\hline 1 Teckel & $\mathrm{F}$ & 11 & Aguda & $\begin{array}{l}\text { Reflexos espinais dimi- } \\
\text { nuídos, tônus muscular } \\
\text { diminuído, paraplegia } \\
\text { sem dor profunda, } \\
\text { dor acima do joelho }\end{array}$ & $\begin{array}{l}\text { Dor acima do joelho; } \\
\text { ausência de pulso } \\
\text { femoral bilateral }\end{array}$ & $36,5^{\circ} \mathrm{C}$ & $36.929,13 \mathrm{UI} / \mathrm{L}$ & Endocardite & $\begin{array}{l}\text { Infartos renais, miosite } \\
\text { necrossupurativa, } \\
\text { cistite fibrinonecrótica }\end{array}$ \\
\hline 3 Teckel & $\mathrm{F}$ & 15 & $\begin{array}{l}\text { Crônica } \\
1 \text { semana }\end{array}$ & $\begin{array}{l}\text { Reflexos espinais } \\
\text { diminuídos, tônus } \\
\text { muscular diminuído, } \\
\text { monoplegia sem } \\
\text { dor profunda e mono- } \\
\text { paresia contralateral }\end{array}$ & $\begin{array}{l}\text { Membros pélvicos } \\
\text { frios; ausência de } \\
\text { pulso femoral } \\
\text { unilateral e filiforme } \\
\text { unilateral }\end{array}$ & $35,2^{\circ} \mathrm{C}$ & 8.679UI/L & $\begin{array}{l}\text { Hemangios- } \\
\text { sarcoma }\end{array}$ & $\begin{array}{l}\text { Êmbolos neoplásicos } \\
\text { rins, baço e fígado }\end{array}$ \\
\hline $5 \mathrm{SRD}^{\mathrm{a}}$ & $\mathrm{F}$ & 12 & Aguda & $\begin{array}{l}\text { Reflexos espinais } \\
\text { diminuídos, tônus } \\
\text { muscular diminuído, } \\
\text { paraplegia sem } \\
\text { dor profunda }\end{array}$ & $\begin{array}{l}\text { Dor acima do joelho; } \\
\text { ausência de pulso } \\
\text { femoral bilateral }\end{array}$ & $36,2^{\circ} \mathrm{C}$ & $10.450 \mathrm{UI} / \mathrm{L}$ & $\begin{array}{c}\text { Não } \\
\text { encontrada }\end{array}$ & $\begin{array}{l}\text { Cistite necro- } \\
\text { hemorrágica, } \\
\text { degeneração e } \\
\text { necrose muscular }\end{array}$ \\
\hline
\end{tabular}

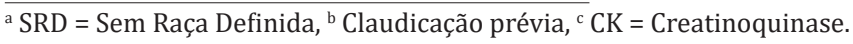




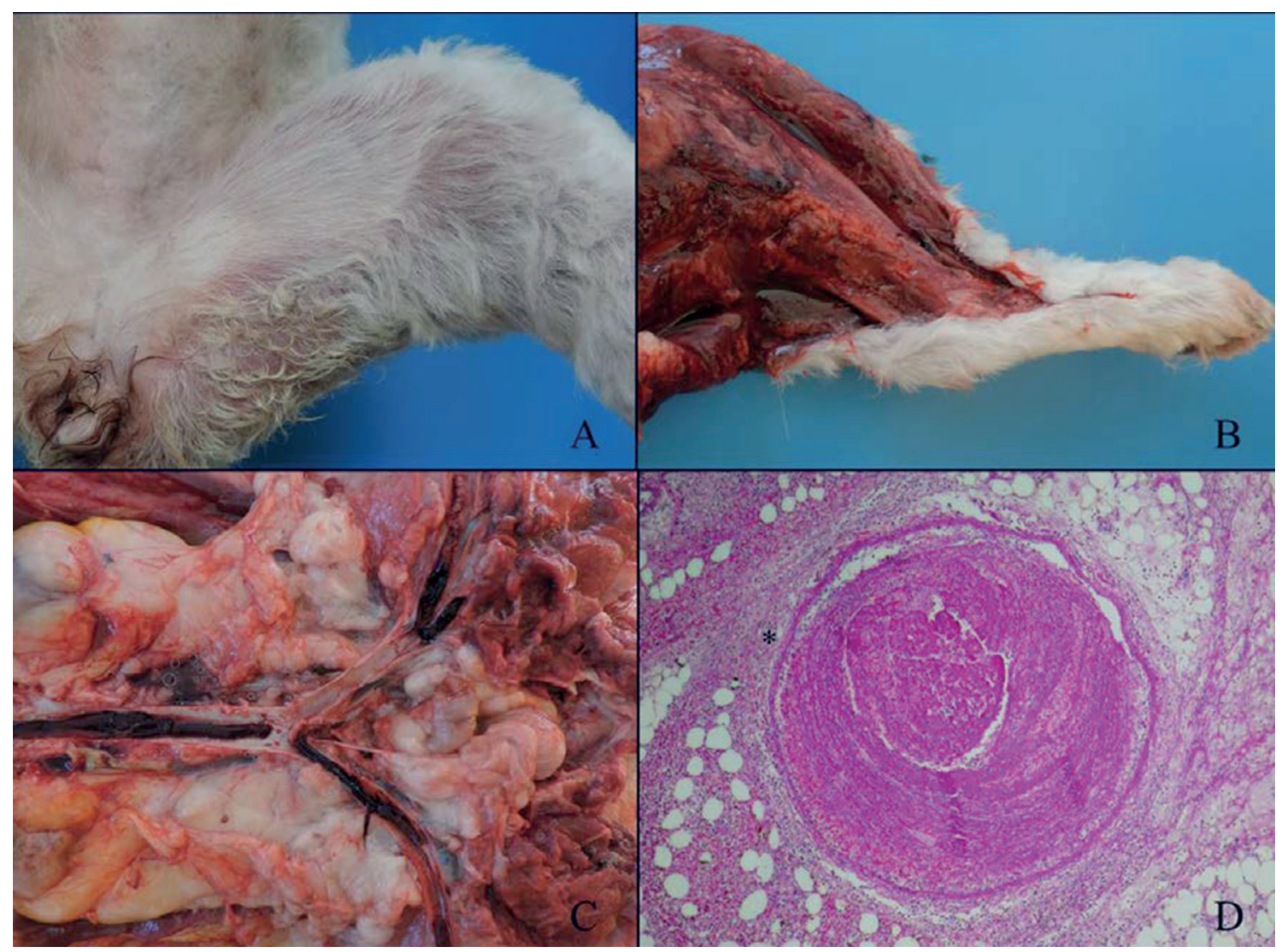

Fig.1 (A) Pele da face medial do membro pélvico esquerdo de um cão com tromboembolismo aórtico. Observe a descoloração do tecido afetado que passa de branco para azulado em uma nítida zona de demarcação. (B) Subcutâneo da face medial do membro pélvico esquerdo de um cão com tromboembolismo aórtico. Intensa necrose hemorrágica caracterizada pelo avermelhamento difuso do tecido. (C) Artérias aorta (porção terminal) e ilíacas externas. Trombo focalmente extenso que oblitera completamente a luz vascular. (D) Ramo da artéria ilíaca externa esquerda. Note o grande trombo de fibrina que oclui completamente o lúmen do vaso sanguíneo e o intenso infiltrado inflamatório perivascular. HE, obj.20x.

(Barger 2015). É um marcador sensível e específico de lesão muscular, pois sua principal fonte são as fibras musculares esqueléticas e cardíacas (Barger 2015), assim, encontra-se aumentada em variados graus nos casos de necrose muscular secundária a miopatias (Podell 2002).

$\mathrm{O}$ aumento da CK pode oscilar em variados graus, mas em alguns casos essa enzima pode estar normal (Boswood et al. 2000, Gonçalves et al. 2008), provavelmente casos crônicos com obstrução parcial do lúmen arterial. Os valores desta enzima ainda não foram associados a prognóstico, porém, quanto mais grave a lesão muscular, mais altos serão os valores desta enzima (Barger 2015) e isso, provavelmente influencia nas condições de bem estar do paciente em casos de tentativa de tratamento.

Na necropsia, três pacientes apresentaram alteração cardíaca, sendo dois de endocardite e um de cardiomiopatia dilatada com presença de trombo focalmente extenso na aurícula esquerda. Sykes et al. (2006) também verificaram que o tromboembolismo arterial foi uma das complicações observadas na endocardite infecciosa e naqueles quadros com claudicação.

Duas pacientes não apresentaram alteração cardíaca à necropsia, assim pode-se inferir que o presente estudo compreende três casos de tromboembolismo secundário à cardiopatia, um caso de tromboembolismo secundário a neoplasma e um caso de trombose. Diversos tipos histológicos de neoplasmas já são descritos como causa de trombose arterial ou tromboembolismo; nesses casos o trombo pode ser formado tanto por células sanguíneas (cão 3, Quadro 1) como por células neoplásicas (Van Winkle et al. 1993, Marcasso et al. 2011, Kim \& Park 2012). O neoplasma pode infiltrar-se em vasos sanguíneos levando à lesão endotelial direta e/ou alteração do fluxo sanguíneo e consequente formação de trombo que, pode se desprender e causar o tromboembolismo (Boswood et al. 2000).

0 hemangiossarcoma observado no presente estudo (cão 3, Quadro 1), pode apresentar formação de coágulos no interior das massas neoplásicas (Flores et al. 2012) que podem embolizar e vir a obstruir a luz de outros vasos sem que haja células neoplásicas no êmbolo. No caso deste neoplasma, sua consistência bastante friável (Flores et al. 2012) facilita também a embolização de células neoplásicas, alterações encontradas também nos vasos renais, esplênicos e hepáticos da paciente em questão.

As cinco pacientes apresentavam trombo focalmente extenso que se estendia da aorta abdominal até a região de bifurcação das artérias ilíacas externas (Fig.1C e D). Em três pacientes, a obstrução do aporte sanguíneo ocasionou necrose muscular e miosite supurativa nos membros pélvicos, demonstrada pela elevação da CK. 
Outros achados de necropsia como infartos renais e esplênicos (Quadro 1) são os locais mais comuns em cães com alteração cardíaca, sobretudo endocardite infecciosa (Sykes et al. 2006, seguidos por miocárdio e encéfalo (cão 4, Quadro 1). A cistite descrita em dois casos aqui abordados (cães 1 e 5, Quadro 1) é decorrente de retenção urinária secundária, provavelmente devido à lesão isquêmica afetar a inervação da vesícula urinária ou músculos detrusores; não se pode descartar a possibilidade da cistite já ser crônica e ter causado a endocardite.

O diagnóstico precoce, quando o paciente ainda não apresenta quadro plégico bilateral talvez seja a chave para maiores taxas de sucesso no tratamento e isso depende tanto da patofisiologia da doença e alterações concomitantes como da habilidade do clínico diagnosticar, associada aos custos da terapia (Konecny 2010). Sendo mais interessante considerar tratamento com fármacos anti-coagulantes em pacientes que ainda apresentam deambulação (Winter et al. 2012), já que em muitos casos ainda pode existir algum fluxo sanguíneo através ou ao redor do trombo (Lake-Bakaar et al. 2012).

Outros métodos diagnósticos para a neuromiopatia isquêmica, além da palpação do pulso arterial já foram descritas incluindo avaliação com termografia (Kim \& Park 2012), ultrassonografia abdominal, angiografia (Van Winkle et al. 1993, Boswood et al. 2000), tomografia computadorizada (Kirberger \& Zambelli 2007), ressonância magnética (Sykes et al. 2006), mensuração da glicose sistêmica e da glicose periférica no membro afetado (Klainbart et al. 2014) ou mesmo a utilização do doppler vascular, como foi realizado em um dos casos do presente estudo. Entretanto, é preciso lembrar que a suspeita clínica inicial que direciona para outros meios diagnósticos, em todos os casos, surge pela ausência ou diminuição do pulso arterial à palpação, um exame simples, rápido e não invasivo. Assim, pode-se considerar este, um exame de extrema importância para todos os pacientes que são encaminhados para avaliação neurológica com queixa de dificuldade para caminhar com os membros pélvicos ou mesmo com paraplegia de evolução aguda ou crônica em que o local da lesão esteja entre os segmentos L4-S3 da medula espinhal. Alteração neste exame pode incluir a neuromiopatia isquêmica na lista de diferenciais, reforçar sua suspeita ou mesmo descartar tal afecção.

0 presente estudo revelou que é importante considerar doenças vasculares no diagnóstico diferencial ao examinar cães com sinais de disfunção neuromuscular ou mesmo com sinais agudos de disfunção neurológica e com intolerância ao exercício por longo período, como salientaram Gonçalves et al. (2008) ou quando apresentam histórico de neoplasma concomitante (Kim \& Park 2012). Essa consideração pode evitar diagnóstico equivocado e consequentemente impedir terapia para outras doenças como as mielopatias compressivas.

Boswood et al. (2000) ainda sugeriram que episódio de tromboembolismo em cães tem menor chance de resultar em eutanásia do que em felinos. Entretanto, no presente estudo, o fator prognóstico desfavorável para a deambulação dos animais interferiu na decisão da eutanásia que ocorreu em $80 \%$ dos casos. Talvez isso fosse reflexo do estado crítico em que alguns cães chegaram para atendimento, já apresentando necrose de coxins e muscular (Quadro 1 e Fig.1) sendo lesão irreversível e incompatível com o bem-estar dos pacientes.

\section{CONCLUSÕES}

A neuromiopatia isquêmica secundária ao tromboembolismo ou trombose aórtica deve ser considerada como diagnóstico diferencial em cães que apresentem claudicação, paraparesia ou paraplegia de início agudo ou crônico, sem distinção de sexo, raça ou idade, mesmo que os sinais clínicos mimetizem disfunção medular do segmento L4-S3.

A avaliação, mesmo que subjetiva, do pulso femoral em cães paréticos ou plégicos pode incitar a suspeita de trombo aórtico como causa dos sinais clínicos nos membros pélvicos, associada a dor muscular acima da articulação do joelho, reflexo cutâneo do tronco e palpação epaxial sem alteração permite direcionamento dos exames complementares.

\section{REFERÊNCIAS}

Barger A.M. 2015. Clinical chemistry, p.95-139. In: Barger A.M. \& Macneill A.L. (Eds), Clinical Pathology and Laboratory Techniques for Veterinary Technicians. Willey Blackwell.

Boswood A., Lamb C.R. \& White R.N. 2000. Aortic and iliac thrombosis in six dogs. J. Small Anim. Pract. 41:109-114.

Dewey C.W. \& Talarico L.R. 2016. Myopathies: Disorders of Skeletal Muscle, p.481-520. In: Dewey, C.W. \& Da Costa R.C. (Eds), Practical Guide of Canine and Feline Neurology, Willey Blackwell.

Diamante G.A.C., Marinho P.V.T., Zani C.C., Bracarense A.P.F.R.L. \& Arias M.V.B. 2016. Tromboembolismo aórtico decorrente de endocardite bacteriana causando neuromiopatia isquêmica em um cão. Acta Scientiae Veterinariae 44(1):1-5.

Flores M.M., Panziera W., Kommers G.D., Irigoyen L.F., Barros C.S.L. \& Fighera R.A. 2012. Aspectos epidemiológicos e anatomopatológicos do hemangiossarcoma em cães: 40 casos (1965-2012). Pesq. Vet. Bras. 32(12):1319-1328.

Goldstein R.E. 2010. Swollen joints and lameness, p.83-87. In: Ettinger J.E. \& Feldman E.C. (Eds), Textbook of Veterinary Internal Medicine. W.B. Saunders, Philadelphia:

Gonçalves R., Penderis J., Chang P., Zoia A., Mosley J. \& Anderson T.J. 2008. Clinical and neurological characteristics of aortic thromboembolism in dogs. J. Small Anim. Pract. 49:178-184.

González F.H.D. \& Da Silva S.C. 2006. Perfil bioquímico sanguíneo, p.313358. In: Ibid. (Eds), Introdução à Bioquímica Clínica Veterinária. UFRGS Editora, Porto Alegre.

Kirberger R.M. \& Zambelli A. 2007. Imaging diagnosis - aortic thromboembolism associated with spirocercosis in a dog. Vet. Radiol. Ultrasound 48(5):418-420.

Kim J.H. \& Park H.M. 2012. Unilateral femoral arterial thrombosis in a dog with malignant mammary gland tumor: clinical and thermographic findings, and successful treatment with local intra-arterial administration of streptokinase. J. Vet. Med. Sci. 74(5):657-661.

Klainbart S., Kelmer E., Vidmayer B., Bdolah-Abram T., Segev G. \& Aroch I. 2014. Peripheral and central venous blood glucose concentrations in dogs and cats with acute arterial thromboembolism. J. Vet. Intern. Med. 28:1513-1519.

Konecny F. 2010. Thromboembolic conditions, aetiology diagnosis and treatment in dogs and cats. Acta Vet. Brno 79:497-508.

Lake-Bakaar G.A., Johnson E.G. \& Griffiths L.G. 2012. Aortic thrombosis in dogs: 31 cases (2000-2010). J. Am. Vet. Med. Assoc. 241(7):910-915. 
Laste N.J. \& Harpster N.K. 1995. A retrospective study of 100 cases of feline distal aortic thromboembolism: 1977-1993. J. Am. Anim. Hosp. Assoc. 31(6):492-500.

Marcasso R.A., Arias M.V.B., Gianini L., Headley S.A. \& Bracarense A.P.F.R.L. 2011. Pheochromocytoma in a dog as a cause of aortic thromboembolism. Braz. J. Vet. Pathol. 4(2):145-149.

Podell M. 2002. Inflammatory myopathies. Vet. Clin. North Am., Small Anim. Pract. 32(1):147-167.

Rosenberg R.D. \& Aird W.C. 1999. Vascular-bed-specific hemostasis and hypercoagulable states. N. Engl. J. Med. 340(20):1555-1564.

Schoeman J.P. 1999. Feline distal aortic thromboembolism: a review of 44 cases (1990-1998). J. Fel. Med. Surg. 1:221-231.

Smith S.A., Tobias A.H., Jacob K.A., Fine D.M. \& Grumbles P.L. 2003. Arterial thromboembolism in cats: acute crisis in 127 cases (1992-2001) and long-term management with low-dose aspirin in 24 cases. J. Vet. Intern. Med. 17(1):73-83.

Smith S.A. \& Tobias A.H. 2004. Feline arterial thromboembolism: an update. Vet. Clin. North Am., Small Anim. Pract. 34:1245-1271.

Sykes J.E., Kittleson M.D., Chomel B.B., MacDonald K.A. \& Pesavento P.A. 2006. Clinicopathologic findings and outcome in dogs with infective endocarditis: 71 cases (1992-2005). J. Am. Vet. Med. Assoc. 228(11):1735-1747.

Thomas W.B. 2000. Initial assessment of patients with neurologic dysfunction. Vet. Clin. North Am., Small Anim. Pract. 30(1):1-24.

Van Winkle T.J., Hackner S.G. \& Liu S.M. 1993. Clinical and pathological features of aortic thromboembolism in 36 dogs. Vet. Emerg. Crit. Care 3(1):13-21.

Winter R.L., Sedacca C.D., Adams A. \& Orton E.C. 2012. Aortic thrombosis in dogs: presentation, therapy, and outcome in 26 cases. J. Vet. Cardiol. 14:333-342. 